\title{
Juegos psicológicos en los procesos de reconciliación'
}

Juan Carlos Valderrama $C^{2}$

Edward Johnn Silva $\mathrm{G}^{3}$

Yasnith Rubiano Ramírez ${ }^{4}$

Maritza Layne ${ }^{5}$

María Alejandra Pinilla $\mathrm{O}^{6}$

Erika Vanegas ${ }^{7}$

María Alejandra Molina $\mathrm{Q}^{8}$

Jackeline Cañas Jaramillo ${ }^{9}$

Bertha Yeniffer Romero $V^{10}$

Alba Lucia Carrillo Avila ${ }^{11}$

Artículo de reflexión recibido el 16/02/2018

y aprobado el 08/03/2018

Cómo citar este artículo:

Valderrama Cardenas, J., Solva Giraldo, E., Rubiano Ramírez, Y., Layne, M., Pinilla Ospina, M., Venegas, E., Molina Quintero, M., Cañas Jaramillo, J., Romero Vargas, B., \& Carrillo Avila, A. (2018). Juegos psicológicos en los procesos de reconciliación. Trans-Pasando Fronteras, (11). 
Miembros del semillero de investigación: Familia, institución y comunidad

\section{Resumen}

Este articulo presenta los resultados preliminares de la fase 1 del proyecto de investigación "La dimensión espiritual en los procesos de perdón y reconciliación en el posconflicto" desarrollado por la Universidad, Luis Amigo y la Corporación Universitaria Minuto de Dios. Se pretende comprender, Desde una perspectiva relacional, a través de las narrativas de los actores del posconflicto los juegos psicológicos/relacionales recurrentes en los procesos de reconciliación; haciendo énfasis en experiencias fallidas y examinando posibles soluciones. El trabajo se apoya en

\footnotetext{
${ }^{1}$ Articulo derivado del proyecto de investigación "la dimensión espiritual en los procesos de perdón y reconciliación en el posconflicto" Universidad Luis Amigo y Corporación Universitaria Minuto de Dios.

2 Docente de investigación, líder del semillero de investigación: Familia, institución y comunidad. Facultad de ciencias humanas y sociales, programa de psicología UNIMINUTO UVD. Correspondencia: juan.valderrama.c@gmail.com

${ }^{3}$ Docente líder de acompañamiento a regiones y del semillero de investigación: Familia, Institución y comunidad. Facultad de ciencias humanas y sociales, programa de psicología UNIMINUTO UVD.: edwardjohnnsilva@hotmail.com

${ }^{4}$ Estudiante miembro del semillero de investigación: Familia, institución y comunidad. Facultad de ciencias humanas y sociales, programa de psicología UNIMINUTO UVD. Correspondencia: yasitarubiano@hotmail.com

${ }^{5}$ Docente investigador, Universidad Luis Amigo. Correspondencia: mlayneco@gmail.com

Estudiante miembro del semillero de investigación: Familia, institución y comunidad. Facultad de ciencias humanas y sociales, programa de psicología UNIMINUTO UVD. malejandra.pinillaos@ gmail.com

${ }^{6}$ Estudiante miembro del semillero de investigación: Familia, institución y comunidad. Facultad de ciencias humanas y sociales, programa de psicología UNIMINUTO UVD. Correspondencia: terik.14@hotmail.com

${ }^{7}$ Estudiante miembro del semillero de investigación: Familia, institución y comunidad. Facultad de ciencias humanas y sociales, programa de psicología UNIMINUTO UVD. mariaalejandramolinaquintero@gmail.com

${ }^{8}$ Estudiante miembro del semillero de investigación: Familia, institución y comunidad. Facultad de ciencias humanas y sociales, programa de psicología UNIMINUTO UVD. jackecj30@gmail.com

${ }^{9}$ Estudiante miembro del semillero de investigación: Familia, institución y comunidad. Facultad de ciencias humanas y sociales, programa de psicología UNIMINUTO UVD.:sdmr12.yr91@gmail.com ${ }^{10}$ Estudiante miembro del semillero de investigación: Familia, institución y comunidad. Facultad de ciencias humanas y sociales, programa de psicología UNIMINUTO UVD.albalu73@gmail.com
} 
las dinámicas definidas en el triángulo dramático (TD) de modo que los relatos describen comportamientos, creencias y emociones que prescriben acciones y estilos de relación; como resultado se identifican dos juegos principales: 1) perdón sin reconciliación, en donde se hace imposible la restauración del tejido social y 2) reconciliación sin perdón, que se caracteriza por un intento frio de cohabitar y de agresiones encubiertas en la comunicación.

Las conclusiones se orientan a cuatro hipótesis de trabajo:

i) los roles asumidos/designados no son estáticos

ii) perdón y reconciliación son procesos interdependientes,

iii) Desconectar los procesos de perdón a los procesos de reconciliación promueve narrativas de desesperanza, iv) los juegos fallidos están basados en la desconfianza.

En ese sentido los retos para la fase 2 de la investigación se encuentran en hacer una descripción de los juegos exitosos en donde perdón y reconciliación se articulen.

Palabras clave: Posconflicto; Reconciliación; Perdón; Victima; Victimario; Ciudadano. 


\section{Psychological games in reconciliation processes}

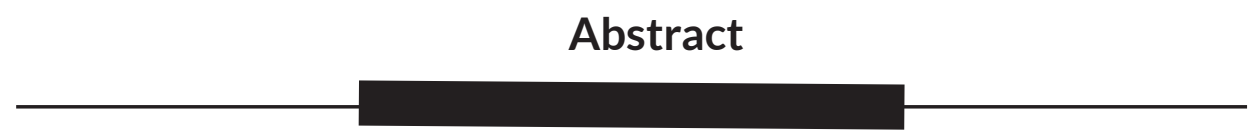

This article presents the preliminary results of the phase 1 of the research project "The spiritual dimension in the processes of forgiveness and reconciliation in the post-conflict" developed by the Universidad Luis Amigo y la Corporación Universitaria Minuto de Dios. From a relational perspective one tries to understand by means of the narratives of the actors of the post-conflict the psychological/relational games appellants in the reconciliation processes, doing emphasis on frustrated experiences and examining possible solutions, the work rests on the dynamics defined in the dramatic triangle (TD) so that the histories describe behaviors, beliefs and emotions that prescribe actions and relation styles; as result two main games are identified: 1) forgiveness without reconciliation, where reconciliation makes impossible to itself the restoration of the social network cohesion and 2) without forgiveness, which is characterized by a cold attempt of living together and covert aggressions in the communication.

The conclusions are faced to four work hypotheses:

1) the assumed/designated rolls are not static,

2) forgiveness and reconciliation are interdependent processes,

3) to Disconnect the forgiveness processes to the reconciliation processes promotes despair narratives and,

4) the frustrated games are based on the suspicion. 
In this sense the challenges for the phase 2 of the investigation are in doing a description of the successful games where forgiveness and reconciliation are articulated.

Key words: Post-conflict; Reconciliation; Forgiveness; Victim; Persecutor; Citizen.

\section{Introducción}

Es de utilidad hacer la primera aproximación a la reconciliación (la espiritualidad y el perdón no son abordados en este artículo) realizando una sencilla distinción con otro concepto que puede ser tomado en ocasiones como un sinónimo. Según la RAE conciliar es poner de acuerdo a dos o más personas, busca hacer compatibles cosas que son opuestas entre si, mientras que reconciliar se refiere a volver a las amistades, y atraer/acordar los ánimos desunidos. Una primera diferencia se encuentra en que cada uno se sitúa en un nivel diferente y, por lo tanto, tienen objetivos particulares, la conciliación trabaja en el nivel de los acuerdos que pretenden ser operativizados en acciones concretas; la reconciliación por otra parte está en el nivel de la relación, y en ese sentido procura una redefinición del vínculo. Desde una perspectiva comunicacional (Watzlawick, Beavin \& Jackson, 1967) se entiende en que los intentos de solución de la conciliación están en el contenido de los acuerdos, y la reconciliación trabaja explícitamente en el nivel relacional de la comunicación.

Una segunda distinción estaría en la gravedad/complejidad del conflicto, en la conciliación aparentemente está en el contenido 
de las diferencias, más no hay una ruptura en la relación; en la reconciliación se entiende que la intensidad del conflicto ha perpetrado una interacción antagónica en donde las agresiones han distanciado significativamente a los participantes.

Reconciliarse con la sombra, es uno de los postulados más icónicos de la psicología analítica propuesta por Carl Jung, en la cual se expone que las personas tienen toda una serie de aspectos indeseados, negados y rechazados de sí mismas; incluso esta sombra puede ser comprendida como una entidad enemiga y antagónica. Groso modo, si las personas no aceptan e integran estos aspectos vendrán implicaciones a nivel de salud mental y de las relaciones sociales (Monbourquette, 1999). Reconciliarse con la sombra significa abandonar las creencias sobre ese enemigo, aceptarlo, reconocer su existencia y luego integrarlo a la vida psíquica. Esta aproximación, aunque construida en lo intrapsíquico, permite considerar algunas nociones básicas, por el momento es útil comprender que la construcción y la cristalización de cualquier relación percibida como negativa tiene un impacto considerable en la calidad de las relaciones sociales.

\section{Los procesos de reconciliación y la salud mental}

El 17 de abril de 2016, en su portal web, Europa Press publica un titular que señala: "La reconciliación tras un conflicto civil empeora la salud psicológica". El estudio realizado en 200 aldeas en los procesos de reconciliación en Sierra Leona señala consecuencias positivas y negativas en la experiencia de estos procesos de reconciliación. En ese orden de ideas, los aspectos positivos se relacionan con un incremento de acciones prosociales, pero con el costo de la salud psicológica de los individuos, puesto que se 
evidencio el aumento de desórdenes como: la depresión, la ansiedad y el estrés postraumático (Cilliers, Oendrilla \& Billal, 2016). El estudio recomienda tener este costo en cuenta en la construcción e implementación de cualquier programa de reconciliación, pues la verdad y el revivir ciertos eventos tienen implicaciones significativas en la salud mental. Por ello, es relevante que los procesos de verdad estén acompañados por profesionales que puedan mitigar las consecuencias negativas de la reconciliación (Cilliers, Oendrilla \& Billal, 2015).

En términos generales se comprende que la reconciliación es el restablecimiento de la relación entre víctima y victimario, teniendo como base un dialogo abierto, y busca la reconstrucción del tejido social (Bueno Cipagauta, 2006). Puede, entonces, entenderse la reconciliación como un proceso complejo que usualmente no es inmediato, ya que se considera como una serie de acciones a largo plazo, y que hacen asunción a la convivencia, en la cual es fundamental el respeto de los derechos y acciones concretas que garanticen dichos derechos (Hinestroza, Saldarriaga \& Berrio, 2016).

\section{La noción de juego psicológico y triangulo dramático}

Berne (1964) definió que las relaciones humanas se dan en transacciones sencillas que conforman juegos complejos; dichos juegos son intercambios complementarios que progresan hacia un resultado más o menos definido y tiene como base motivaciones ocultas de los participantes de la interacción. En ese sentido el triángulo dramático (TD) propuesto por Karpman (1973) consiste en un juego en donde hay tres roles/actores: 
1) el perseguidor, que está legitimado para dominar e imponer, y sus emociones negativas son justificadas, es crítico con los demás, y el objetivo de su juego se relaciona con la venganza.

2) el salvador, quien desea proteger, su posición es dominante pero encubierto y, ayuda así no se haya solicitado su apoyo, su juego consiste en sentirse superior a los otros.

3) la víctima, que se muestra desamparada y vulnerable, sin embargo, es un desamparo agresivo, en el que suele culpar a alguien -el perseguidor-y delegar sus acciones a otros -el salvador-, de modo que puede asumir actitudes defensivas (Liras, 2015).

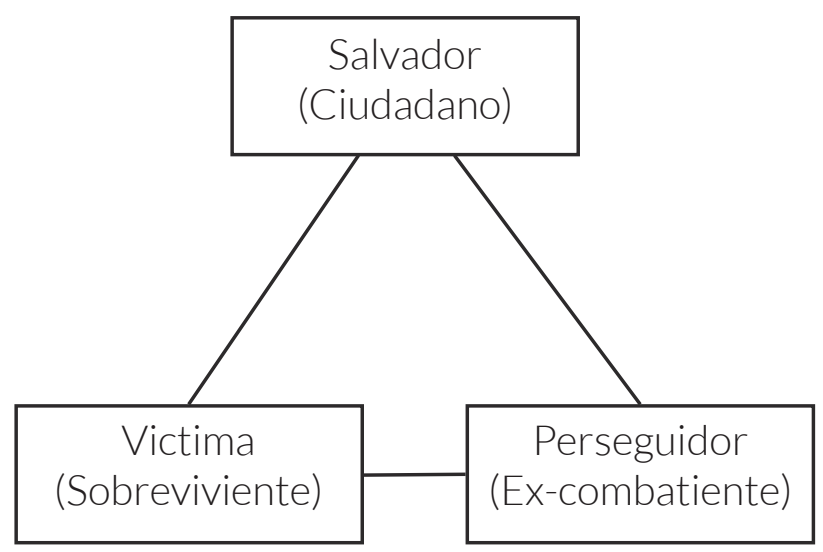

\section{Metodología}

La fase 1 del proyecto de investigación comprende lo siguiente:

1. Construcción del marco teórico sobre espiritualidad, perdón y reconciliación. 
2. Elaboración de la primera propuesta de instrumento para realizar entrevistas semiestructuradas.

3. Realizar una prueba piloto de la entrevista semiestructurada.

4. Analizar las narrativas de las personas entrevistadas teniendo como base las categorías de análisis planteadas.

5. Plantear hipótesis de trabajo respecto a posibles juegos relacionales que caracterizan el posconflicto.

La fase 2 del proyecto se desarrollará durante el año 2018, por lo tanto, no se tiene presente en este artículo.

\section{Instrumento}

Entrevista semiestructurada la cual cuenta con las siguientes categorías de análisis:

- Espiritualidad

- $\quad$ Experiencias de perdón

- Experiencias de reconciliación

\section{Resultados y discusión}

Los procesos de reconciliación no responden a una formula mecánica, ya que, desde la perspectiva de la construcción de paz, se propone pasar de conocer el evento a reconocer a las personas,

${ }^{12}$ Se realizaron dos entrevistas con personas que han vivido el conflicto armado en Colombia, en donde retroalimentaron el instrumento y su pertinencia. 
a través de un marco de referencia centrado en las relaciones, que posibilita los espacios de encuentro para conversar sobre el pasado, imaginar el futuro y reconstruir el presente mediante nuevas percepciones y experiencias compartidas (Lederach, 2007).

\section{Juegos psicológicos/relacionales en que participan los actores del posconflicto}

Las lógicas de interacción enmarcadas en la guerra, se fundamentan en la pretensión de eliminar al contrario, confirmando el mito hobbesiano que señala que para defender la vida se debe competir contra los otros (Silva, 2016). Es de especial interés en este proyecto comprender dos juegos que se presentan en el conflicto armado y en el posconflicto: 1) Perdón sin reconciliación (Tabla 1) y 2) Reconciliación sin perdón (Tabla 2).

\begin{tabular}{|l|l|}
\hline Rol & Juegos y narrativas dominantes \\
\hline Perseguidor & $\begin{array}{l}\text { Se relaciona con los otros desde una critica encubierta: “...hay muchos que no entienden el } \\
\text { tema del conflicto, se dejan influenciar por lo que dicen otros...” }\end{array}$ \\
\hline Victima & $\begin{array}{l}\text { Renuncia a la venganza, pero no considera compartir con el excombatiente: “... en mi corazón } \\
\text { ya no hay odio, pero no podría compartir con esa gente, no podría tomarme un café con...” }\end{array}$ \\
\hline Salvador & $\begin{array}{l}\text { Desea asumir el cuidado de la víctima desde la distancia, y no permite que el excombatiente } \\
\text { se integre: “...esa pobre gente, todo lo que han sufrido, y estos otros todo el daño que han hecho } \\
\text { ¿ypretenden estar como si nada?...” }\end{array}$ \\
\hline
\end{tabular}

Tabla 1. Juegos y narrativas dominantes cuando hay perdón sin reconciliación.

El ciudadano/salvador no ha sufrido directamente los efectos de la guerra, pero muestra preocupación por las necesidades y el dolor de las víctimas del conflicto armando. Sin embargo, con la intención de ayuda, brinda un apoyo no solicitado, asumiendo una posición de superioridad que reduce la percepción de capacidad de las personas que su ubican en una posición de víctima. Dicho 
planteamiento requiere una redefinición de los roles, en la lógica de los procesos democráticos que respeten y reconozcan la pluralidad y las diferencias de las poblaciones vulnerables, ya que la dinámica del conflicto debe superar las tensiones ente víctimas y victimarios que paralizan el agenciamiento personal y colectivo (López, 2011).

\begin{tabular}{|l|l|}
\hline Rol & Juegos y narrativas dominantes \\
\hline Perseguidor & $\begin{array}{l}\text { La relación se muestra agresiva y con fronteras rígidas en el tejido social: "sino perdono y } \\
\text { sino me perdonan, esto no va a cambiar..." }\end{array}$ \\
\hline Victima & $\begin{array}{l}\text { La experiencia de co-habitar se caracteriza por el temor: "... si uno de esos xxx fuera mi vecino, } \\
\text { ¿Quién me garantiza que voy a estar segura?, ¿Quién podría vivir así? ...." }\end{array}$ \\
\hline Salvador & $\begin{array}{l}\text { La relación es definida desde la desconfianza: "... no puedo imaginarme eso, seguiría pasando } \\
\text { lo mismo, las matanzas, los maltratos, solo que ahora seria a la vista de todos..." }\end{array}$ \\
\hline
\end{tabular}

Tabla 2. Juegos y narrativas dominantes cuando hay reconciliación sin perdón.

El juego de la víctima, suele girar alrededor de la acusación y la visión centrada en el pasado, delegando la responsabilidad de sus acciones en otros actores que propician la configuración de un sistema de alta dependencia y baja autonomía (Edwards, 2011). El riesgo en esta interacción, se presenta cuando se instala la categoría de victima a la identidad personal. El planteamiento del TD, señala que el victimario en el contexto del conflicto armado, participa de una dinámica de polarización en la que defiende una postura ideológica de verdad absoluta, con la que justifica su necesidad de hacer entrar en razón a los demás actores, paradójicamente a través de lemas sustentados en discursos de paz y acciones relacionadas con la violencia. 


\section{Conclusiones}

Se presentan las conclusiones a modo de cuatro hipótesis de trabajo:

\begin{tabular}{|l|l|}
\hline Hipótesis 1 & Los roles no son rígidos. \\
\hline Hipótesis 2 & Perdón y reconciliación son procesos interdependientes \\
\hline Hipótesis 3 & $\begin{array}{l}\text { Desconectar los procesos de perdón a los procesos de reconciliación promueve narrativas de } \\
\text { desesperanza }\end{array}$ \\
\hline Hipótesis 4 & Todos los juegos están basados en la desconfianza \\
\hline
\end{tabular}

Tabla 3. Conclusiones

\section{Referencias}

Berne, E. (1964). Juegos en que participamos, Psicología de las relaciones humanas. México D.F., México: Editorial Diana.

Bueno Cipagauta, M. (2006). La reconciliación como un proceso socio-político. Aproximaciones teóricas. Reflexión Política, junio, 6478. Recuperado de: http://www.redalyc.org/html/110/11001506/

Cilliers J., Dube, O., y Siddqi, B. (2016). Reconciling after civil conflict increases social capital but decreases individual well-being. Science, 352(6287), 787-794. Recuperado de: http://science. sciencemag.org/content/352/6287/787

Cilliers J., Dube, O., y Siddqi, B. (2015). Can the Wounds of War be Healed?: Experimental Evidence on Reconciliation in Sierra Leone. Recuperado de: http://odube.net/papers/Reconciliation.pdf 
Edwards, G. (2011). El triángulo dramático de Karpman: Cómo trascender los roles de perseguidor, salvador o víctima. Establece relaciones personales saludables. Madrid, España: GAIA Ediciones.

Hinestroza, I. M., Osorio, L. A. T., Saldarriaga, M. G. M., \& Berrio, S. M. A. (2016). Perdón, convivencia y reconciliación en el proceso de paz, desde una mirada psicológica. Poiésis, (31), 245-256.

Karpman, S. B. (1973). 1972 Eric Berne memorial scientific award lecture. Transactional Analysis Bulletin, 3(1), 73-77. Recuperado de: http://journals.sagepub.com/doi/abs/10.1177/03621 5377300300118?journalCode=taxa

Lederach, J. (2007). Construyendo la paz. Reconciliación sostenible en sociedades divididas. Bilbao, España: Editorial Gernika Gogoratuz.

Liras, S. M. (2015). How to Break Free of the Drama Triangle \& Victim Consciousness. Clínica, 6(2), 129-133. Recuperado de: http:// www.copmadrid.org/webcopm/publicaciones/clinicacontemporanea/cc2015v6n2a6.pdf

López, W. (2011). Medios de Comunicación, Conflicto y Paz: sobre el enmarcamiento psicosocial del conflicto sociopolítico y la paz en Colombia (Tesis doctoral) Universidad de Santiago de Compostela, España.

Monbourquette, J. (1999). Reconciliarse con la propia sombra: el lado oscuro de la persona (Vol. 56). Santander, España: Editorial Sal Terrae.

Press, E. (2016). La reconciliación tras un conflicto civil empeora la salud psicológica. Salud: Infosalus. Recuperado de: http:// 
www.infosalus.com/salud-investigacion/noticia-reconciliacionconflicto-civil-empeora-salud-psicologica-20160517082632. html

Silva, E. (2016). "Las trampas del lenguaje". El observador. Cajicá, 12 de diciembre, 2016. Recuperado de: http://elobservador. $\mathrm{co} /$ ?s=las+trampas+del+lenguaje

Watzlawick, P. Beavin, J. \& Jackson, D. (1967). Teoría de la comunicación humana. Barcelona, España: Ed Herder. 